\title{
On renormalizability of the massless Thirring model
}

\author{
H. Bozkaya*, A. N. Ivanov ${ }^{\dagger \ddagger}$, M. Pitschmann ${ }^{\S}$
}

October 15, 2018

\author{
Atominstitut der Österreichischen Universitäten, Technische Universität Wien, \\ Wiedner Hauptstrasse 8-10, A-1040 Wien, Österreich
}

\begin{abstract}
We discuss the renormalizability of the massless Thirring model in terms of the causal fermion Green functions and correlation functions of left-right fermion densities. We obtain the most general expressions for the causal two-point Green function and correlation function of left-right fermion densities with dynamical dimensions of fermion fields, parameterised by two parameters. The region of variation of these parameters is constrained by the positive definiteness of the norms of the wave functions of the states related to components of the fermion vector current. We show that the dynamical dimensions of fermion fields calculated for causal Green functions and correlation functions of left-right fermion densities can be made equal. This implies the renormalizability of the massless Thirring model in the sense that the ultra-violet cut-off dependence, appearing in the causal fermion Green functions and correlation functions of left-right fermion densities, can be removed by renormalization of the wave function of the massless Thirring fermion fields only.

PACS: 11.10.Gh, 11.10.Kk, 11.10.Lm, 11.30.Rd
\end{abstract}

\footnotetext{
${ }^{*} \mathrm{E}-$ mail: hidir@kph.tuwien.ac.at, Tel.: +43-1-58801-14262, Fax: +43-1-58801-14299

${ }^{\dagger} \mathrm{E}-\mathrm{mail}$ : ivanov@kph.tuwien.ac.at, Tel.: +43-1-58801-14261, Fax: +43-1-58801-14299

${ }_{\ddagger}^{\ddagger}$ Permanent Address: State Polytechnic University, Department of Nuclear Physics, 195251 St. Petersburg, Russian Federation

${ }^{\S}$ E-mail: pitschmann@kph.tuwien.ac.at, Tel.: +43-1-58801-14263, Fax: +43-1-58801-14299
} 


\section{Introduction}

The massless Thirring model [1] is an exactly solvable quantum field theoretic model of fermions with a non-trivial four-fermion interaction in 1+1-dimensional space-time defined by the Lagrangian invariant under the chiral group $U_{\mathrm{V}}(1) \times U_{\mathrm{A}}(1)$

$$
\mathcal{L}_{\mathrm{Th}}(x)=\bar{\psi}(x) i \gamma^{\mu} \partial_{\mu} \psi(x)-\frac{1}{2} g \bar{\psi}(x) \gamma^{\mu} \psi(x) \bar{\psi}(x) \gamma_{\mu} \psi(x)
$$

where $\psi(x)$ is a massless Dirac fermion field and $g$ is a dimensionless coupling constant that can be both positive and negative.

A solution of the Thirring model assumes a development of a procedure for the calculation of any correlation function [2]-[8. As has been shown by Hagen [4] and Klaiber [5], the correlation functions of massless Thirring fermion fields can be parameterised by one arbitrary parameter. In Hagen's notation this parameter is $\xi$. Below we show that the correlation functions in the massless Thirring model can be parameterised by two parameters (see Appendix A). This confirms the results obtained by Harada et al. [9] (see also [10, 11]) for the chiral Schwinger model. In our notation these parameters are $\bar{\xi}$ and $\bar{\eta}$. The region of variation of these parameters is restricted by the condition for the norms of the wave functions of the states related to the components of the fermion vector current to be positive (see Appendix B). For $\bar{\eta}=1$ the parameter $\bar{\xi}$ is equal to Hagen's parameter $\bar{\xi}=\xi$. The parameters $\bar{\xi}$ and $\bar{\eta}$ we use for the analysis of the non-perturbative renormalizability of the massless Thirring model in the sense that a dependence of any correlation function on the ultra-violet cut-off $\Lambda$ can be removed by the renormalization of the wave function of Thirring fermion fields only. We show that independence of any correlation function of an ultra-violet cut-off exists only if the dynamical dimensions of Thirring fermion fields, calculated from different correlation functions, are equal. We would like to remind that for the known solutions of the massless Thirring model [2] - 8] the dynamical dimensions of massless Thirring fermion fields, calculated from causal Green functions and left-right correlation functions, are different. The existence of different dynamical dimensions of Thirring fermion fields obtained from different correlation functions has been regarded by Jackiw as a problem of 1+1-dimensional quantum field theories 12 .

The paper is organised as follows. In Section 2 we define the generating functional of correlation functions in the massless Thirring model. In Section 3 we calculate the twopoint causal Green function and the dynamical dimension of massless Thirring fermion fields $d_{\bar{\psi} \psi}(g)$, parameterised by two parameters $\bar{\xi}$ and $\bar{\eta}$. In Section 4 we calculate the twopoint correlation function of the left-right fermion densities and the dynamical dimension of massless Thirring fermion fields $d_{(\bar{\psi} \psi)^{2}}(g)$ in dependence on $\bar{\xi}$ and $\bar{\eta}$. We show that the dynamical dimensions $d_{\bar{\psi} \psi}(g)$ and $d_{(\bar{\psi} \psi)^{2}}(g)$ can be made equal $d_{(\bar{\psi} \psi)^{2}}(g)=d_{\bar{\psi} \psi}(g)$. This indicates that the massless Thirring model is renormalizable in the sense that the dependence of the causal Green functions and correlation functions of left-right fermion densities on the ultra-violet cut-off can be removed by the renormalization of the wave function of massless Thirring fermion fields. In Section 5 we corroborate the validity of this assertion within the standard renormalization procedure [13]. In the Conclusion we discuss the obtained results. In Appendix A we show that the $\operatorname{determinant} \operatorname{Det}(i \hat{\partial}+\hat{A})$, where $A_{\mu}$ is an external vector field, can be parameterised by two parameters. For this aim we

calculate the vacuum expectation value of the vector current and show that the ambiguous 
parameterisation of the determinant $\operatorname{Det}(i \hat{\partial}+\hat{A})$ is fully caused by the regularization procedure [9]-11]. In Appendix B we analyse the constraints on the parameters $\bar{\xi}$ and $\bar{\eta}$ imposed by the positive definiteness of the norms of the wave functions of the states related to the components of the vector fermion current. We show that the positive definiteness of these norms does not prohibit the possibility for the dynamical dimensions of massless Thirring fermion fields to be equal. According to the equivalence of the massive Thirring model to the sine-Gordon model [14], the constraints on the parameters $\bar{\eta}$ and $\bar{\xi}$ together with the requirement of the non-perturbative renormalizability of the massless Thirring model lead to the strongly coupled sine-Gordon field with the coupling constant $\beta^{2} \sim 8 \pi$. A behaviour and renormalizability of the sine-Gordon model for the coupling constants $\beta^{2} \sim 8 \pi$ has been investigated in [15].

\section{Generating functional of correlation functions}

The generating functional of vacuum expectation values of products of massless Thirring fermion fields, i.e. correlation functions, is defined by

$$
\begin{aligned}
Z_{\mathrm{Th}}[J, \bar{J}]= & \int \mathcal{D} \psi \mathcal{D} \bar{\psi} \exp i \int d^{2} x\left[\bar{\psi}(x) i \gamma^{\mu} \partial_{\mu} \psi(x)-\frac{1}{2} g \bar{\psi}(x) \gamma^{\mu} \psi(x) \bar{\psi}(x)\right. \\
& +\bar{\psi}(x) J(x)+\bar{J}(x) \psi(x)]
\end{aligned}
$$

It can be represented also as follows

$$
Z_{\mathrm{Th}}[J, \bar{J}]=\left.\exp \left\{\frac{i}{2} g \int d^{2} x \frac{\delta}{\delta A_{\mu}(x)} \frac{\delta}{\delta A^{\mu}(x)}\right\} Z_{\mathrm{Th}}^{(0)}[A ; J, \bar{J}]\right|_{A=0},
$$

where we have denoted

$$
\begin{aligned}
Z_{\mathrm{Th}}^{(0)}[A ; J, \bar{J}]= & \int \mathcal{D} \psi \mathcal{D} \bar{\psi} \exp i \int d^{2} x\left[\bar{\psi}(x) i \gamma^{\mu} \partial_{\mu} \psi(x)+\bar{\psi}(x) \gamma^{\mu} \psi(x) A_{\mu}(x)\right. \\
& +\bar{\psi}(x) J(x)+\bar{J}(x) \psi(x)] .
\end{aligned}
$$

The functional $Z_{\mathrm{Th}}^{(0)}[A ; J, \bar{J}]$ is a generating functional of vacuum expectation values of products of massless fermion fields of the massless Schwinger model coupled to an external vector field $A_{\mu}(x)$ [16. The integration over fermion fields can be carried out explicitly and we get

$$
Z_{\mathrm{Th}}^{(0)}[A ; J, \bar{J}]=\operatorname{Det}(i \hat{\partial}+\hat{A}) \exp \left\{i \iint d^{2} x d^{2} y \bar{J}(x) G(x, y)_{A} J(y)\right\}
$$

where $G(x, y)_{A}$ is a two-point causal fermion Green function obeying the equation

$$
i \gamma^{\mu}\left(\frac{\partial}{\partial x^{\mu}}-i A_{\mu}(x)\right) G(x, y)_{A}=-\delta^{(2)}(x-y)
$$

As has been shown in the Appendix A, the functional determinant $\operatorname{Det}(i \hat{\partial}+\hat{A})$ can be parameterised by two parameters

$$
\operatorname{Det}(i \hat{\partial}+\hat{A})=\exp \left\{\frac{i}{2} \iint d^{2} x d^{2} y A_{\mu}(x) D^{\mu \nu}(x-y) A_{\nu}(y)\right\}
$$


where we have denoted

$$
D^{\mu \nu}(x-y)=\frac{\bar{\xi}}{\pi} g^{\mu \nu} \delta^{(2)}(x-y)-\frac{\bar{\eta}}{\pi} \frac{\partial}{\partial x_{\mu}} \frac{\partial}{\partial x_{\nu}} \Delta(x-y ; \mu) .
$$

Here $\bar{\xi}$ and $\bar{\eta}$ are two parameters, $g^{\mu \nu}$ is the metric tensor and $\Delta(x-y ; \mu)$ is the causal two-point Green function of a free massless (pseudo)scalar field

$$
i \Delta(x-y ; \mu)=\frac{1}{4 \pi} \ln \left[-\mu^{2}(x-y)^{2}+i 0\right] .
$$

It obeys the equation $\square_{x} \Delta(x-y ; \mu)=\delta^{(2)}(x-y)$, where $\mu$ is an infrared cut-off.

The appearance of two parameters is caused by dependence of the calculation of the determinant $\operatorname{Det}(i \hat{\partial}+\hat{A})$ on the regularization procedure [9]-11. In Appendix B we find the constraint on the region of variation of these parameters imposed by the positive definiteness of the norms of the wave functions of the states related to the components of the fermion vector current. The parameters $\bar{\xi}$ and $\bar{\eta}$ are related to Hagen's parameter $\xi$ as $\bar{\xi}=\xi$ and $\bar{\eta}=1$.

The solution of the equation (2.5) is equal to

$$
\begin{aligned}
& G(x, y)_{A}=G_{0}(x-y) \\
& \times \exp \left\{-i\left(g^{\alpha \beta}-\varepsilon^{\alpha \beta} \gamma^{5}\right) \int d^{2} z \frac{\partial}{\partial z^{\alpha}}[\Delta(x-z ; \mu)-\Delta(y-z ; \mu)] A_{\beta}(z)\right\},
\end{aligned}
$$

where $\varepsilon^{\alpha \beta}$ is the antisymmetric tensor defined by $\varepsilon^{01}=1$ and $G_{0}(x-y)$ is the Green function of a free massless fermion field

$$
G_{0}(x-y)=i \gamma^{\mu} \frac{\partial}{\partial x^{\mu}} \Delta(x-y ; \mu)=\frac{1}{2 \pi} \frac{\gamma^{\mu}(x-y)_{\mu}}{(x-y)^{2}-i 0}
$$

satisfying the equation $i \gamma^{\mu} \partial_{\mu} G_{0}(x-y)=-\delta^{(2)}(x-y)$.

Any correlation function of the massless Thirring fermion fields can be defined by functional derivatives of the generating functional (2.1) and calculated in terms of the two-point Green functions $G(x, y)_{A}$ and $\Delta(x-y ; \mu)$. Below we calculate the casual twopoint Green function $G(x, y)$ and the correlation function $C(x, y)$ of the left-right fermion densities defined by

$$
\begin{aligned}
G(x, y) & =i\langle 0|\mathrm{~T}(\psi(x) \bar{\psi}(y))| 0\rangle=\left.\frac{1}{i} \frac{\delta}{\delta \bar{J}(x)} \frac{\delta}{\delta J(y)} Z_{\mathrm{Th}}[J, \bar{J}]\right|_{J=\bar{J}=0}, \\
C(x, y) & =\left\langle 0\left|\mathrm{~T}\left(\bar{\psi}(x)\left(\frac{1-\gamma^{5}}{2}\right) \psi(x) \bar{\psi}(y)\left(\frac{1+\gamma^{5}}{2}\right) \psi(y)\right)\right| 0\right\rangle= \\
& =\left.\frac{1}{i} \frac{\delta}{\delta J(x)}\left(\frac{1-\gamma^{5}}{2}\right) \frac{1}{i} \frac{\delta}{\delta \bar{J}(x)} \frac{1}{i} \frac{\delta}{\delta J(y)}\left(\frac{1+\gamma^{5}}{2}\right) \frac{1}{i} \frac{\delta}{\delta \bar{J}(y)} Z_{\mathrm{Th}}[J, \bar{J}]\right|_{J=\bar{J}=0},
\end{aligned}
$$

where $\mathrm{T}$ is the time-ordering operator. The main aim of the investigation of these correlation functions is in the calculation of the dynamical dimensions of the massless Thirring fermion fields and the analysis of the possibility to make them equal [12]. 


\section{Two-point causal Green function $G(x, y)$}

In terms of the generating functional (2.1) the two-point Green function $G(x, y)$ is defined by

$$
\begin{aligned}
& G(x, y)=\left.\frac{1}{i} \frac{\delta}{\delta \bar{J}(x)} \frac{\delta}{\delta J(y)} Z_{\mathrm{Th}}[J, \bar{J}]\right|_{J=\bar{J}=0}=\exp \left\{\frac{i}{2} g \int d^{2} z \frac{\delta}{\delta A_{\mu}(z)} \frac{\delta}{\delta A^{\mu}(z)}\right\} \\
& \quad \times\left.\exp \left\{\frac{i}{2} \iint d^{2} z_{1} d^{2} z_{2} A_{\lambda}\left(z_{1}\right) D^{\lambda \varphi}\left(z_{1}-z_{2}\right) A_{\varphi}\left(z_{2}\right)\right\} G(x, y)_{A}\right|_{A=0} .
\end{aligned}
$$

The calculation of the r.h.s. of (3.1) reduces to the calculation of the path integral

$$
\begin{aligned}
G(x, y)= & \frac{1}{2 \pi} \frac{\gamma^{\mu}(x-y)_{\mu}}{(x-y)^{2}-i 0} \int \mathcal{D}^{2} u \exp \left\{-\frac{i}{2} \int d^{2} z u_{\mu}(z) u^{\mu}(z)\right. \\
& -\frac{i}{2} g \iint d^{2} z_{1} d^{2} z_{2} u_{\mu}\left(z_{1}\right) D^{\mu \nu}\left(z_{1}-z_{2}\right) u_{\nu}\left(z_{2}\right)+\sqrt{g}\left(g^{\alpha \beta}-\varepsilon^{\alpha \beta} \gamma^{5}\right) \\
& \left.\times \int d^{2} z \frac{\partial}{\partial z^{\alpha}}[\Delta(x-z ; \mu)-\Delta(y-z ; \mu)] u_{\beta}(z)\right\} .
\end{aligned}
$$

Symbolically the r.h.s. of (3.2) can be written as

$$
\begin{aligned}
& G(x, y)=\frac{1}{2 \pi} \frac{\gamma^{\mu}(x-y)_{\mu}}{(x-y)^{2}-i 0} \\
& \times \int \mathcal{D}^{2} u \exp \left\{-\frac{i}{2} u(1+g D) u+\sqrt{g} \partial\left(\Delta_{x}-\Delta_{y}\right) u-\sqrt{g} \gamma^{5} \partial\left(\Delta_{x}-\Delta_{y}\right) \varepsilon u\right\} .
\end{aligned}
$$

The integration over $u$ can be carried out by quadratic extension. This yields

$$
\begin{aligned}
G(x, y)= & \frac{1}{2 \pi} \frac{\gamma^{\mu}(x-y)_{\mu}}{(x-y)^{2}-i 0} \exp \left\{-\frac{i}{2} g \partial\left(\Delta_{x}-\Delta_{y}\right) \frac{1}{1+g D} \partial\left(\Delta_{x}-\Delta_{y}\right)\right. \\
& \left.+\frac{i}{2} g \partial\left(\Delta_{x}-\Delta_{y}\right) \varepsilon \frac{1}{1+g D} \varepsilon \partial\left(\Delta_{x}-\Delta_{y}\right)\right\} .
\end{aligned}
$$

For the subsequent calculation we have to construct the matrix $(1+g D)^{-1}$. The matrix $(1+g D)$ has the following elements

$$
(1+g D)^{\mu \alpha}(x, z)=\left(1+\bar{\xi} \frac{g}{\pi}\right) g^{\mu \alpha} \delta^{(2)}(x-z)-\bar{\eta} \frac{g}{\pi} \frac{\partial}{\partial x_{\mu}} \frac{\partial}{\partial x_{\alpha}} \Delta(x-z ; \mu) .
$$

The elements of the matrix $(1+g D)^{-1}$ we define as

$$
\left((1+g D)^{-1}\right)_{\alpha \nu}(z, y)=A g_{\alpha \nu} \delta^{(2)}(z-y)+B \frac{\partial}{\partial z^{\alpha}} \frac{\partial}{\partial z^{\nu}} \Delta(z-y ; \mu) .
$$

The matrices $(1+g D)$ and $(1+g D)^{-1}$ should obey the condition

$$
\int d^{2} z(1+g D)^{\mu \alpha}(x, z)\left((1+g D)^{-1}\right)_{\alpha \nu}(z, y)=g_{\nu}^{\mu} \delta^{(2)}(x-y) .
$$


This gives

$$
\begin{aligned}
\left((1+g D)^{-1}\right)_{\alpha \nu}(z, y)= & \frac{g_{\alpha \nu}}{1+\bar{\xi} \frac{g}{\pi}} \delta^{(2)}(z-y) \\
& +\frac{g}{\pi} \frac{\bar{\eta}}{\left(1+\bar{\xi} \frac{g}{\pi}\right)\left(1+(\bar{\xi}-\bar{\eta}) \frac{g}{\pi}\right)} \frac{\partial}{\partial z^{\alpha}} \frac{\partial}{\partial z^{\nu}} \Delta(z-y ; \mu) .
\end{aligned}
$$

Using (3.8) we obtain

$$
\begin{aligned}
-\frac{i}{2} g \partial\left(\Delta_{x}-\Delta_{y}\right) \frac{1}{1+g D} \partial\left(\Delta_{x}-\Delta_{y}\right) & =\frac{g}{1+(\bar{\xi}-\bar{\eta}) \frac{g}{\pi}}[i \Delta(0 ; \mu)-i \Delta(x-y ; \mu)], \\
+\frac{i}{2} g \partial\left(\Delta_{x}-\Delta_{y}\right) \varepsilon \frac{1}{1+g D} \varepsilon \partial\left(\Delta_{x}-\Delta_{y}\right) & =-\frac{g}{1+\bar{\xi} \frac{g}{\pi}}[i \Delta(0 ; \mu)-i \Delta(x-y ; \mu)],
\end{aligned}
$$

where $i \Delta(0 ; \mu)$ is equal to

$$
i \Delta(0 ; \mu)=-\frac{1}{4 \pi} \ln \left(\frac{\Lambda^{2}}{\mu^{2}}\right) .
$$

Thus, the two-point Green function reads

$$
\begin{aligned}
G(x, y) & =\frac{1}{2 \pi} \frac{\gamma^{\mu}(x-y)_{\mu}}{(x-y)^{2}-i 0} e^{4 \pi d_{\bar{\psi} \psi}(g)[i \Delta(0 ; \mu)-i \Delta(x-y ; \mu)]} \\
& =-\frac{\Lambda^{2}}{2 \pi} \frac{\gamma^{\mu}(x-y)_{\mu}}{-\Lambda^{2}(x-y)^{2}+i 0}\left[-\Lambda^{2}(x-y)^{2}+i 0\right]^{-d_{(\bar{\psi} \psi)}(g)} \\
& =\Lambda G\left(d_{\bar{\psi} \psi}(g) ; \Lambda x, \Lambda y\right)
\end{aligned}
$$

where $d_{\bar{\psi} \psi}(g)$ is a dynamical dimension of the Thirring fermion field defined by [12]

$$
d_{\bar{\psi} \psi}(g)=\frac{g^{2}}{4 \pi^{2}} \frac{\bar{\eta}}{\left(1+\bar{\xi} \frac{g}{\pi}\right)\left(1+(\bar{\xi}-\bar{\eta}) \frac{g}{\pi}\right)} .
$$

Now we are proceeding to the calculation of the correlation function $C(x, y)$.

\section{Two-point correlation function $C(x, y)$}

According to Eq.(2.11), the two-point correlation function $C(x, y)$ of the left-right fermion densities is defined by

$$
\begin{aligned}
& C(x, y)=\left.\frac{1}{i} \frac{\delta}{\delta J(x)}\left(\frac{1-\gamma^{5}}{2}\right) \frac{1}{i} \frac{\delta}{\delta \bar{J}(x)} \frac{1}{i} \frac{\delta}{\delta J(y)}\left(\frac{1+\gamma^{5}}{2}\right) \frac{1}{i} \frac{\delta}{\delta \bar{J}(y)} Z_{\mathrm{Th}}[J, \bar{J}]\right|_{J=\bar{J}=0}= \\
& =-\exp \left\{\frac{i}{2} g \int d^{2} z \frac{\delta}{\delta A_{\mu}(z)} \frac{\delta}{\delta A^{\mu}(z)}\right\} \exp \left\{\frac{i}{2} \iint d^{2} z_{1} d^{2} z_{2} A_{\lambda}\left(z_{1}\right) D^{\lambda \varphi}\left(z_{1}-z_{2}\right) A_{\varphi}\left(z_{2}\right)\right\} \\
& \times\left.\operatorname{tr}\left\{G(y, x)_{A}\left(\frac{1-\gamma^{5}}{2}\right) G(x, y)_{A}\left(\frac{1+\gamma^{5}}{2}\right)\right\}\right|_{A=0} .
\end{aligned}
$$


This reduces to the calculation of the path integral

$$
\begin{aligned}
C(x, y)= & \frac{1}{4 \pi^{2}} \frac{1}{(x-y)^{2}-i 0} \\
& \times \int \mathcal{D}^{2} u \exp \left\{-\frac{i}{2} u(1+g D) u-2 \sqrt{g} \partial\left(\Delta_{x}-\Delta_{y}\right) \varepsilon u\right\}= \\
= & \frac{1}{4 \pi^{2}} \frac{1}{(x-y)^{2}-i 0} \exp \left\{2 i g \partial\left(\Delta_{x}-\Delta_{y}\right) \varepsilon \frac{1}{1+g D} \varepsilon \partial\left(\Delta_{x}-\Delta_{y}\right)\right\}
\end{aligned}
$$

The result is

$$
\begin{aligned}
C(x, y) & =\frac{1}{4 \pi^{2}} \frac{1}{(x-y)^{2}-i 0} e^{8 \pi d_{(\bar{\psi} \psi)^{2}}[i \Delta(0 ; \mu)-i \Delta(x-y ; \mu)]}= \\
& =-\frac{\Lambda^{2}}{4 \pi^{2}} \frac{1}{-\Lambda^{2}(x-y)^{2}+i 0}\left[-\Lambda^{2}(x-y)^{2}+i 0\right]^{-2 d_{(\bar{\psi} \psi)^{2}}}= \\
& =\Lambda^{2} C\left(d_{(\bar{\psi} \psi)^{2}}(g) ; \Lambda x, \Lambda y\right) .
\end{aligned}
$$

The dynamical dimension $d_{(\bar{\psi} \psi)^{2}}$ is equal to

$$
d_{(\bar{\psi} \psi)^{2}}(g)=-\frac{g}{2 \pi} \frac{1}{1+\bar{\xi} \frac{g}{\pi}} .
$$

For $\bar{\xi}$ and $\bar{\eta}$, restricted only by the constraint caused by the positive definiteness of the norms of the wave functions of the states related to the components of the fermion vector current (see Appendix B), the dynamical dimensions of the massless Thirring model, calculated for the two-point causal Green function (3.12) and the correlation function of the left-right fermion densities (4.4), are not equal. According to Jackiw [12], this is a problem of quantum field theories in 1+1-dimensional space-time. However, equating $d_{(\bar{\psi} \psi)^{2}}(g)$ and $d_{\bar{\psi} \psi}(g)$ we get the constraint on the parameter $\bar{\eta}$

$$
\bar{\eta}=\frac{2 \pi}{g}\left(1+\bar{\xi} \frac{g}{\pi}\right)
$$

As has been shown in Appendix B, the constraint on the region of variation of parameters $\bar{\xi}$ and $\bar{\eta}$, imposed by the positive definiteness of the norms of the wave functions of the states related to the components of the vector current, does not prevent from the equality of dynamical dimensions $d_{(\bar{\psi} \psi)^{2}}(g)=d_{\bar{\psi} \psi}(g)$.

This indicates that the massless Thirring model is renormalizable. The dependence on the ultra-violet cut-off $\Lambda$ can be removed by the renormalization of the wave functions of Thirring fermion fields either for the $2 n$-point Green functions $G\left(x_{1}, \ldots, x_{n} ; y_{1}, \ldots, y_{n}\right)$ or for the $2 n$-point correlation functions $C\left(x_{1}, \ldots, x_{n} ; y_{1}, \ldots, y_{n}\right)$ of the left-right fermion densities. The dynamical dimension of the Thirring fermion fields is equal to $d_{\psi}(g)=$ $d_{(\bar{\psi} \psi)^{2}}(g)$ defined by Eq.(4.4).

\section{Non-perturbative renormalization}

According to the standard procedure of renormalization in quantum field theory [13] the renormalizability of the massless Thirring model should be understood as a possibility to 
remove all ultra-violet and infrared divergences by renormalization of the wave function of the massless Thirring fermion field $\psi(x)$ and the coupling constant $g$.

Let us rewrite the Lagrangian (1.1) in terms of bare quantities

$$
\mathcal{L}_{\mathrm{Th}}(x)=\bar{\psi}_{0}(x) i \gamma^{\mu} \partial_{\mu} \psi_{0}(x)-\frac{1}{2} g_{0} \bar{\psi}_{0}(x) \gamma^{\mu} \psi_{0}(x) \bar{\psi}_{0}(x) \gamma_{\mu} \psi_{0}(x),
$$

where $\psi_{0}(x), \bar{\psi}_{0}(x)$ are bare fermionic field operators and $g_{0}$ is a bare coupling constant.

The renormalized Lagrangian $\mathcal{L}(x)$ of the massless Thirring model should then read 13

$$
\begin{aligned}
\mathcal{L}_{\mathrm{Th}}(x)= & \bar{\psi}(x) i \gamma^{\mu} \partial_{\mu} \psi(x)-\frac{1}{2} g \bar{\psi}(x) \gamma^{\mu} \psi(x) \bar{\psi}(x) \gamma_{\mu} \psi(x) \\
& +\left(Z_{2}-1\right) \bar{\psi}(x) i \gamma^{\mu} \partial_{\mu} \psi(x)-\frac{1}{2} g\left(Z_{1}-1\right) \bar{\psi}(x) \gamma^{\mu} \psi(x) \bar{\psi}(x) \gamma_{\mu} \psi(x)= \\
= & Z_{2} \bar{\psi}(x) i \gamma^{\mu} \partial_{\mu} \psi(x)-\frac{1}{2} g Z_{1} \bar{\psi}(x) \gamma^{\mu} \psi(x) \bar{\psi}(x) \gamma_{\mu} \psi(x),
\end{aligned}
$$

where $Z_{1}$ and $Z_{2}$ are the renormalization constants of the coupling constant and the wave function of the fermion field.

The renormalized fermionic field operator $\psi(x)$ and the coupling constant $g$ are related to bare quantities by the relations [13]

$$
\begin{aligned}
\psi_{0}(x) & =Z_{2}^{1 / 2} \psi(x), \\
g_{0} & =Z_{1} Z_{2}^{-2} g .
\end{aligned}
$$

For the correlation functions of massless Thirring fermions the renormalizability of the massless Thirring model means the possibility to replace the infrared cut-off $\mu$ and the ultra-violet cut-off $\Lambda$ by a finite scale $M$ by means of the renormalization constants $Z_{1}$ and $Z_{2}$.

According to the general theory of renormalization [13], the renormalization constants $Z_{1}$ and $Z_{2}$ depend on the renormalized quantities $g$, the infrared scale $\mu$, the ultra-violet scale $\Lambda$ and the finite scale $M$. As has been shown above the Green functions and left-right fermion density correlation functions do not depend on the infrared cut-off. Therefore, we can omit it. This defines the renormalization constants as follows

$$
\begin{aligned}
& Z_{1}=Z_{1}(g, M ; \Lambda), \\
& Z_{2}=Z_{2}(g, M ; \Lambda) .
\end{aligned}
$$

For the analysis of the feasibility of the replacement $\Lambda \rightarrow M$ it is convenient to introduce the following notations

$$
\begin{aligned}
& G^{(0)}\left(x_{1}, \ldots, x_{n} ; y_{1}, \ldots, y_{n}\right)=\Lambda^{n} G^{(0)}\left(d_{(\bar{\psi} \psi)}\left(g_{0}\right) ; \Lambda x_{1}, \ldots, \Lambda x_{n} ; \Lambda y_{1}, \ldots, \Lambda y_{n}\right) \\
& C^{(0)}\left(x_{1}, \ldots, x_{n} ; y_{1}, \ldots, y_{n}\right)=\Lambda^{2 n} C^{(0)}\left(d_{(\bar{\psi} \psi)^{2}}\left(g_{0}\right) ; \Lambda x_{1}, \ldots, \Lambda x_{n} ; \Lambda y_{1}, \ldots, \Lambda y_{n}\right) .
\end{aligned}
$$

The transition to a finite scale $M$ changes the functions (5.5) as follows

$$
G^{(0)}\left(x_{1}, \ldots, x_{n} ; y_{1}, \ldots, y_{n}\right)=
$$




$$
\begin{aligned}
& \quad=\left(\frac{\Lambda}{M}\right)^{-2 n d_{(\bar{\psi} \psi)}(g)} M^{n} G^{(0)}\left(d_{(\bar{\psi} \psi)}\left(g_{0}\right) ; M x_{1}, \ldots, M x_{n} ; M y_{1}, \ldots, M y_{n}\right), \\
& C^{(0)}\left(x_{1}, \ldots, x_{n} ; y_{1}, \ldots, y_{n}\right)= \\
& \quad=\left(\frac{\Lambda}{M}\right)^{-4 n d_{(\bar{\psi} \psi)^{2}}(g)} M^{2 n} C^{(0)}\left(d_{(\bar{\psi} \psi)^{2}}\left(g_{0}\right) ; M x_{1}, \ldots, M x_{n} ; M y_{1}, \ldots, M y_{n}\right)(5.6)
\end{aligned}
$$

The renormalized correlation functions are related to the bare ones by the relations [13]:

$$
\begin{aligned}
& G^{(r)}\left(x_{1}, \ldots, x_{n} ; y_{1}, \ldots, y_{n}\right)=Z_{2}^{-n} G^{(0)}\left(x_{1}, \ldots, x_{n} ; y_{1}, \ldots, y_{n}\right)= \\
& =Z_{2}^{-n}\left(\frac{\Lambda}{M}\right)^{-2 n d_{(\bar{\psi} \psi)}(g)} M^{n} G^{(0)}\left(d_{(\bar{\psi} \psi)}\left(Z_{1} Z_{2}^{-2} g\right) ; M x_{1}, \ldots, M x_{n} ; M y_{1}, \ldots, M y_{n}\right), \\
& C^{(r)}\left(x_{1}, \ldots, x_{n} ; y_{1}, \ldots, y_{n}\right)=Z_{2}^{-2 n} C^{(0)}\left(x_{1}, \ldots, x_{n} ; y_{1}, \ldots, y_{n}\right)= \\
& =Z_{2}^{-2 n}\left(\frac{\Lambda}{M}\right)^{-4 n d_{(\bar{\psi} \psi)^{2}}(g)} M^{2 n} C^{(0)}\left(d_{(\bar{\psi} \psi)^{2}}\left(Z_{1} Z_{2}^{-2} g\right) ; M x_{1}, \ldots, M x_{n} ; M y_{1}, \ldots, M y_{n}\right) .
\end{aligned}
$$

Renormalizability demands the relations

$$
\begin{aligned}
& G^{(r)}\left(x_{1}, \ldots, x_{n} ; y_{1}, \ldots, y_{n}\right)=M^{n} G^{(r)}\left(d_{(\bar{\psi} \psi)}(g) ; M x_{1}, \ldots, M x_{n} ; M y_{1}, \ldots, M y_{n}\right) \\
& C^{(r)}\left(x_{1}, \ldots, x_{n} ; y_{1}, \ldots, y_{n}\right)=M^{2 n} C^{(r)}\left(d_{(\bar{\psi} \psi)^{2}}(g) ; M x_{1}, \ldots, M x_{n} ; M y_{1}, \ldots, M y_{n}\right)
\end{aligned}
$$

which impose constraints on the dynamical dimensions and renormalization constants

$$
\begin{aligned}
d_{(\bar{\psi} \psi)}(g) & =d_{(\bar{\psi} \psi)}\left(Z_{1} Z_{2}^{-2} g\right), \\
d_{(\bar{\psi} \psi)^{2}}(g) & =d_{(\bar{\psi} \psi)^{2}}\left(Z_{1} Z_{2}^{-2} g\right)
\end{aligned}
$$

and

$$
Z_{2}^{-1}\left(\frac{\Lambda}{M}\right)^{-2 d_{(\bar{\psi} \psi)}(g)}=Z_{2}^{-1}\left(\frac{\Lambda}{M}\right)^{-2 d_{(\bar{\psi} \psi)^{2}}(g)}=1 .
$$

The constraints (5.9) on the dynamical dimensions are fulfilled only if the renormalization constants are related by

$$
Z_{1}=Z_{2}^{2}
$$

The important consequence of this relation is that the coupling constant $g$ of the massless Thirring model is unrenormalized, i.e.

$$
g_{0}=g \text {. }
$$

This also implies that the Gell-Mann-Low $\beta$-function, defined by [13]

$$
M \frac{d g}{d M}=\beta(g, M)
$$


should vanish, since $g$ is equal to $g_{0}$, which does not depend on $M$, i.e. $\beta(g, M)=0$. Our observation concerning the unrenormalizability of the coupling constant, $g_{0}=g$, is supported by the results obtained in [17 for the massive Thirring model.

The constraint (5.10) is fulfilled only for $d_{(\bar{\psi} \psi)}(g)=d_{(\bar{\psi} \psi)^{2}}(g)$. In this case the dependence of the $2 n$-point causal Green functions and the $2 n$-point correlation functions of left-right fermion densities on the ultra-violet cut-off $\Lambda$ can be simultaneously removed by renormalization of the wave function of the massless Thirring fermion fields. This means the massless Thirring model is non-perturbative renormalizable.

\section{Conclusion}

We have found the most general expressions for the causal two-point Green function and the two-point correlation function of left-right fermion densities with dynamical dimensions parameterised by two parameters. The region of variation of these parameters is restricted by the positive definiteness of the norms of the wave functions of the states related to the components of the fermion vector current (see Appendix B).

Our expressions incorporate those obtained by Hagen, Klaiber and within the pathintegral approach [4-8]. Indeed, for Hagen's parameterisation of the functional determinant with the parameters $\bar{\xi}=\xi$ and $\bar{\eta}=1$ the dynamical dimensions $d_{\bar{\psi} \psi}(g)$ and $d_{(\bar{\psi} \psi)^{2}}(g)$ take the form

$$
d_{\bar{\psi} \psi}(g)=\frac{g^{2}}{2 \pi^{2}} \frac{1}{\left(1+\xi \frac{g}{\pi}\right)\left(1-\eta \frac{g}{\pi}\right)} \quad, \quad d_{(\bar{\psi} \psi)^{2}}(g)=-\frac{g}{2 \pi} \frac{1}{1+\xi \frac{g}{\pi}} .
$$

For $\xi=1$ we get

$$
d_{\bar{\psi} \psi}(g)=\frac{g^{2}}{2 \pi^{2}} \frac{1}{1+\frac{g}{\pi}} \quad, \quad d_{(\bar{\psi} \psi)^{2}}(g)=-\frac{g}{2 \pi} \frac{1}{1+\frac{g}{\pi}} .
$$

These are dynamical dimensions of the Green functions and correlation functions of leftright fermion densities obtained by Klaiber [5] and within the path-integral approach [6] -8 .

We have shown that dynamical dimensions $d_{\bar{\psi} \psi}(g)$ and $d_{(\bar{\psi} \psi)^{2}}(g)$ can be made equal. This fixes the parameter $\bar{\eta}$ in terms of the parameter $\bar{\xi}$ and gives the dynamical dimension of the massless Thirring fermion fields equal to

$$
d_{\bar{\psi} \psi}(g)=d_{(\bar{\psi} \psi)^{2}}(g)=d_{\psi}(g)=-\frac{g}{2 \pi} \frac{1}{1+\bar{\xi} \frac{g}{\pi}} .
$$

As has been pointed out by Jackiw [12, the inequality of dynamical dimensions of fermion fields obtained from different correlation functions is the problem of $1+1$-dimensional quantum field theories. The equality of the dynamical dimensions $d_{\bar{\psi} \psi}(g)$ and $d_{(\bar{\psi} \psi)^{2}}(g)$ is not suppressed by the positive definiteness of the norms of the wave functions of the states related to the components of the vector currents. A positive definiteness of the norms of the wave functions of these states imposes some constraints on the region of 
variation of the parameters $\bar{\eta}$ and $\bar{\xi}$, demanding the parameter $1+\bar{\xi} g / \pi$ to be negative, i.e. $1+\bar{\xi} g / \pi<0$.

This makes the massless Thirring model renormalizable in the sense that the dependence of correlation functions of Thirring fermion fields on the ultra-violet cut-off can be removed by renormalization of the wave function of Thirring fermion fields only. We have corroborated this assertion within the standard renormalization procedure.

From the constraint $-g(1+\bar{\xi} g / \pi)>0$ there follows that the coupling constant $\beta^{2}$ of the sine-Gordon model is of order $\beta^{2} \sim 8 \pi$. A behaviour and renormalizability of the sine-Gordon model for the coupling constants $\beta^{2} \sim 8 \pi$ has been investigated in [15].

We are grateful to Manfried Faber for numerous helpful discussions.

\section{Appendix A: On the parameterisation of the func- tional determinant $\operatorname{Det}(i \hat{\partial}+\hat{A})$}

The result of the calculation of the functional determinant $\operatorname{Det}(i \hat{\partial}+\hat{A})$ is related to the vacuum expectation value $\left\langle j^{\mu}(x)\right\rangle$ of the vector current $j^{\mu}(x)=\bar{\psi}(x) \gamma^{\mu} \psi(x)$. Using (2.4), the vacuum expectation value of the vector current can be defined by

$$
\begin{aligned}
\left\langle j^{\mu}(x)\right\rangle & =\left.\frac{1}{i} \frac{\delta}{\delta A_{\mu}(x)} \ln Z_{\mathrm{th}}^{(0)}[A, J, \bar{J}]\right|_{\bar{J}=J=0}= \\
& =\frac{1}{i} \frac{\delta}{\delta A_{\mu}(x)} \ln \operatorname{Det}(i \hat{\partial}+\hat{A})=\int d^{2} y D^{\mu \nu}(x-y) A_{\nu}(y)
\end{aligned}
$$

where $D^{\mu \nu}(x-y)$ is given by (2.7) and parameterised by two parameters $\bar{\xi}$ and $\bar{\eta}$. Hence, the calculation of the vacuum expectation value of the vector current should show how many parameters one can use for the parameterisation of the Green function $D^{\mu \nu}(x-y)$ or the functional determinant $\operatorname{Det}(i \hat{\partial}+\hat{A})$ as well. According to Hagen $\left[4,\left\langle j^{\mu}(x)\right\rangle\right.$ can be determined by

$$
\left\langle j^{\mu}(x)\right\rangle=\lim _{y \rightarrow x} \operatorname{tr}\left\{i G(x, y)_{A} \gamma^{\mu} \exp i \int_{x}^{y} d z^{\nu}\left(a A_{\nu}(z)+b \gamma^{5} A_{5 \nu}(z)\right)\right\},
$$

where $a$ and $b$ are parameters and $A_{5}^{\nu}(z)=-\varepsilon^{\nu \beta} A_{\beta}(z)$. The fermion Green function $G(y, x)_{A}$ is given by (2.9). The requirement of covariance relates the parameters $a$ and $b$. This provides the parameterisation of the functional determinant $\operatorname{Det}(i \hat{\partial}+\hat{A})$ by one parameter. In Hagen's notation this is the parameter $\xi$.

In order to show that the functional determinant $\operatorname{Det}(i \hat{\partial}+\hat{A})$ can be parameterised by two parameters we propose to define the vacuum expectation value of the vector current $(\mathrm{A}-2)$ as follows

$$
\begin{aligned}
& \left\langle j^{\mu}(x)\right\rangle=\lim _{y \rightarrow x} \operatorname{tr}\left\{i G ( x , y ) _ { A } \gamma ^ { \mu } \operatorname { e x p } i \int _ { x } ^ { y } d z ^ { \nu } \left(a A_{\nu}(z)+b \gamma^{5} A_{5 \nu}(z)\right.\right. \\
& \left.\left.+c \int d^{2} t \frac{\partial}{\partial t^{\nu}} \frac{\partial}{\partial t_{\beta}} \Delta(z-t ; \mu) A_{\beta}(t)+d \gamma^{5} \int d^{2} t \frac{\partial}{\partial t^{\nu}} \frac{\partial}{\partial t_{\beta}} \Delta(z-t ; \mu) A_{5 \beta}(t)\right)\right\}
\end{aligned}
$$

where $c$ and $d$ are additional parameters and $\Delta(z-t ; \mu)$ is determined by (2.8). Under the gauge transformation $A_{\nu} \rightarrow A_{\nu}^{\prime}=A_{\nu}+\partial_{\nu} \phi$ the third term in (A-3) behaves like the first one, whereas the fourth one is gauge invariant. 
The vacuum expectation value of the vector current can be transcribed into the form

$$
\begin{aligned}
& \left\langle j^{\mu}(x)\right\rangle=\lim _{y \rightarrow x} \frac{i}{2 \pi} \frac{(x-y)_{\rho}}{(x-y)^{2}-i 0} \operatorname{tr}\left\{\gamma ^ { \rho } \operatorname { e x p } \left(-i\left(g^{\alpha \beta}-\varepsilon^{\alpha \beta} \gamma^{5}\right)\right.\right. \\
& \left.\times \int d^{2} z \frac{\partial}{\partial z^{\alpha}}[\Delta(x-z ; \mu)-\Delta(y-z ; \mu)] A_{\beta}(z)\right) \gamma^{\mu} \exp i \int_{x}^{y} d z^{\nu}\left(a A_{\nu}(z)+b \gamma^{5} A_{5 \nu}(z)\right. \\
& \left.\left.+c \int d^{2} t \frac{\partial}{\partial t^{\nu}} \frac{\partial}{\partial t_{\beta}} \Delta(z-t ; \mu) A_{\beta}(t)+d \gamma^{5} \int d^{2} t \frac{\partial}{\partial t^{\nu}} \frac{\partial}{\partial t_{\beta}} \Delta(z-t ; \mu) A_{5 \beta}(t)\right)\right\} .
\end{aligned}
$$

For the calculation of the r.h.s. of (A-4) we apply the spatial-point-slitting technique. We set $y^{0}=x^{0}$ and $y^{1}=x^{1} \pm \epsilon$, taking the limit $\epsilon \rightarrow 0$. This gives

$$
\begin{aligned}
\left\langle j^{\mu}(x)\right\rangle= & \lim _{\epsilon \rightarrow 0} \frac{i}{2 \pi} \frac{1}{\mp \epsilon} \operatorname{tr}\left\{\gamma^{1}\left[1 \mp i \epsilon\left(g^{\alpha \beta}-\varepsilon^{\alpha \beta} \gamma^{5}\right) \frac{\partial}{\partial x^{1}} \frac{\partial}{\partial x^{\alpha}} \int d^{2} z \Delta(x-z ; \mu) A_{\beta}(z)\right]\right. \\
& \times \gamma^{\mu}\left[1 \pm i \epsilon\left(a A_{1}(x)+b \gamma^{5} A_{51}(x)+c \int d^{2} t \frac{\partial}{\partial t^{1}} \frac{\partial}{\partial t_{\beta}} \Delta(x-t ; \mu) A_{\beta}(t)\right.\right. \\
& \left.\left.\left.+d \gamma^{5} \int d^{2} t \frac{\partial}{\partial t^{1}} \frac{\partial}{\partial t_{\beta}} \Delta(x-t ; \mu) A_{5 \beta}(t)\right)\right]\right\}= \\
= & \mp \lim _{\epsilon \rightarrow 0} \frac{i g^{1 \mu}}{\pi \epsilon}+\lim _{\epsilon \rightarrow 0} \frac{i}{2 \pi \epsilon}\left[i \epsilon\left(2 g^{1 \mu} g^{\alpha \beta}+2 \varepsilon^{1 \mu} \varepsilon^{\alpha \beta}\right) \frac{\partial}{\partial x^{1}} \frac{\partial}{\partial x^{\alpha}} \int d^{2} z \Delta(x-z ; \mu) A_{\beta}(z)\right. \\
& \mp i \epsilon\left(2 a g^{1 \mu} A_{1}(x)+2 b \varepsilon^{1 \mu} A_{51}(x)+2 c g^{1 \mu} \int d^{2} t \frac{\partial}{\partial t^{1}} \frac{\partial}{\partial t_{\beta}} \Delta(x-t ; \mu) A_{\beta}(t)\right. \\
& \left.\left.+2 d \varepsilon^{1 \mu} \int d^{2} t \frac{\partial}{\partial t^{1}} \frac{\partial}{\partial t_{\beta}} \Delta(x-t ; \mu) A_{5 \beta}(t)\right)\right],
\end{aligned}
$$

Taking the symmetric limit we get

$$
\begin{aligned}
\left\langle j^{\mu}(x)\right\rangle= & \frac{1}{\pi}\left[-\left(g^{1 \mu} g^{\alpha \beta}+\varepsilon^{1 \mu} \varepsilon^{\alpha \beta}\right) \frac{\partial}{\partial x^{1}} \frac{\partial}{\partial x^{\alpha}} \int d^{2} z \Delta(x-z ; \mu) A_{\beta}(z)\right. \\
& +\left(a g^{1 \mu} A_{1}(x)+b \varepsilon^{1 \mu} A_{51}(x)+c g^{1 \mu} \int d^{2} t \frac{\partial}{\partial t^{1}} \frac{\partial}{\partial t_{\beta}} \Delta(x-t ; \mu) A_{\beta}(t)\right. \\
& \left.\left.+d \varepsilon^{1 \mu} \int d^{2} t \frac{\partial}{\partial t^{1}} \frac{\partial}{\partial t_{\beta}} \Delta(x-t ; \mu) A_{5 \beta}(t)\right)\right] .
\end{aligned}
$$

The components of the current are equal to

$$
\begin{aligned}
\left\langle j^{0}(x)\right\rangle= & \frac{1}{\pi} \varepsilon^{\alpha \beta} \frac{\partial}{\partial x^{1}} \frac{\partial}{\partial x^{\alpha}} \int d^{2} z \Delta(x-z ; \mu) A_{\beta}(z) \\
& -\frac{b}{\pi} A_{51}(x)-\frac{d}{\pi} \int d^{2} t \frac{\partial}{\partial t^{1}} \frac{\partial}{\partial t_{\beta}} \Delta(x-t ; \mu) A_{5 \beta}(t), \\
\left\langle j^{1}(x)\right\rangle= & \frac{1}{\pi} \frac{\partial}{\partial x^{1}} \frac{\partial}{\partial x_{\alpha}} \int d^{2} z \Delta(x-z ; \mu) A_{\alpha}(z) \\
& -\frac{a}{\pi} A_{1}(x)-\frac{c}{\pi} \int d^{2} t \frac{\partial}{\partial t^{1}} \frac{\partial}{\partial t_{\beta}} \Delta(x-t ; \mu) A_{\beta}(t) .
\end{aligned}
$$

Using $A_{5 \mu}=-\varepsilon_{\mu \nu} A^{\nu}$ and $\square \Delta(x-y ; \mu)=\delta^{(2)}(x-y)$ the zero component can be transcribed into the form

$$
\left\langle j^{0}(x)\right\rangle=\frac{1}{\pi} \frac{\partial}{\partial x^{1}} \frac{\partial}{\partial x^{0}} \int d^{2} z \Delta(x-z ; \mu) A_{1}(z)-\frac{1}{\pi} \frac{\partial}{\partial x^{1}} \frac{\partial}{\partial x^{1}} \int d^{2} z \Delta(x-z ; \mu) A_{0}(z)
$$




$$
\begin{aligned}
& +\frac{b}{\pi} A^{0}(x)+\frac{d}{\pi} \int d^{2} t \frac{\partial}{\partial t^{1}} \frac{\partial}{\partial t^{0}} \Delta(x-t ; \mu) A_{1}(t) \\
& -\frac{d}{\pi} \int d^{2} t \frac{\partial}{\partial t^{1}} \frac{\partial}{\partial t^{1}} \Delta(x-t ; \mu) A_{0}(t)= \\
& =-\frac{1}{\pi} \frac{\partial}{\partial x^{0}} \frac{\partial}{\partial x_{\mu}} \int d^{2} z \Delta(x-z ; \mu) A_{\mu}(z)+\frac{d}{\pi} A^{0}(x) \\
& +\frac{b+1}{\pi} A^{0}(x)-\frac{d}{\pi} \int d^{2} t \frac{\partial}{\partial t^{0}} \frac{\partial}{\partial t_{\mu}} \Delta(x-t ; \mu) A_{\mu}(t)= \\
& =-\frac{1+d}{\pi} \frac{\partial}{\partial x_{0}} \frac{\partial}{\partial x_{\mu}} \int d^{2} z \Delta(x-z ; \mu) A_{\mu}(z)+\frac{b+d+1}{\pi} A^{0}(x) .
\end{aligned}
$$

Comparing the time component with the spatial one, given by

$$
\left\langle j^{1}(x)\right\rangle=\frac{c-1}{\pi} \frac{\partial}{\partial x_{1}} \frac{\partial}{\partial x_{\mu}} \int d^{2} z \Delta(x-z ; \mu) A_{\mu}(z)+\frac{a}{\pi} A^{1}(x),
$$

we obtain that the covariance of the vacuum expectation value of the vector current takes place for $c=-d$ and $b+d+1=a$ only.

Thus, the vacuum expectation value of the vector current is

$$
\begin{aligned}
\left\langle j^{\mu}(x)\right\rangle & =\frac{\bar{\xi}}{\pi} A^{\mu}(x)-\frac{\bar{\eta}}{\pi} \frac{\partial}{\partial x_{\mu}} \frac{\partial}{\partial x_{\nu}} \int d^{2} z \Delta(x-z ; \mu) A_{\nu}(z) \\
& =\int d^{2} y D^{\mu \nu}(x-y) A_{\nu}(y),
\end{aligned}
$$

where $\bar{\eta}$ and $\bar{\xi}$ are parameters related to the parameters $a, b, c$ and $d$ as $\bar{\xi}=a$ and $\bar{\eta}=1-c$. The vacuum expectation value of the vector current, given by (A-10), supports the possibility to parameterise the functional determinant $\operatorname{Det}(i \hat{\partial}+\hat{A})$ as well as the Green function $D^{\mu \nu}(x-y)$ by two parameters (2.7).

\section{Appendix B: Constraints on the parameters $\bar{\xi}$ and $\bar{\eta}$ from the norms of the wave functions of the states related to the components of the vector current}

The dependence of the functional determinant $\operatorname{Det}(i \hat{\partial}+\hat{A})$ on two parameters leads to the dependence of the two-point correlation function $\left\langle 0\left|\mathrm{~T}\left(j^{\mu}(x) j^{\nu}(y)\right)\right| 0\right\rangle$ on these parameters. Following Johnson [2], for the vacuum expectation value $\left\langle 0\left|\mathrm{~T}\left(j^{\mu}(x) j^{\nu}(y)\right)\right| 0\right\rangle$ we get

$$
\begin{aligned}
i\left\langle 0\left|\mathrm{~T}\left(j^{\mu}(x) j^{\nu}(y)\right)\right| 0\right\rangle= & -\frac{\bar{\eta}}{\pi} \frac{1}{\left(1+\bar{\xi} \frac{g}{\pi}\right)\left(1+(\bar{\xi}-\bar{\eta}) \frac{g}{\pi}\right)} \frac{\partial}{\partial x_{\mu}} \frac{\partial}{\partial x_{\nu}} \Delta(x-y ; \mu) \\
& +\frac{\bar{\eta}}{\pi} \frac{1}{\left(1+\bar{\xi} \frac{g}{\pi}\right)\left(1+(\bar{\xi}-\bar{\eta}) \frac{g}{\pi}\right)} g^{\mu 0} g^{\nu 0} \delta^{(2)}(x-y) .
\end{aligned}
$$


This gives the following expressions for the vacuum expectation values $\left\langle 0\left|j^{0}(x) j^{0}(y)\right| 0\right\rangle$ and $\left\langle 0\left|j^{1}(x) j^{1}(y)\right| 0\right\rangle$

$$
\begin{aligned}
\left\langle 0\left|j^{0}(x) j^{0}(y)\right| 0\right\rangle & =-\frac{\bar{\eta}}{\pi} \frac{1}{\left(1+\bar{\xi} \frac{g}{\pi}\right)\left(1+(\bar{\xi}-\bar{\eta}) \frac{g}{\pi}\right)}\left(\frac{\partial}{\partial x^{1}}\right)^{2} D^{(+)}(x-y) \\
\left\langle 0\left|j^{1}(x) j^{1}(y)\right| 0\right\rangle & =-\frac{\bar{\eta}}{\pi} \frac{1}{\left(1+\bar{\xi} \frac{g}{\pi}\right)\left(1+(\bar{\xi}-\bar{\eta}) \frac{g}{\pi}\right)}\left(\frac{\partial}{\partial x^{1}}\right)^{2} D^{(+)}(x-y),
\end{aligned}
$$

where $D^{( \pm)}(x-y)$ are the Wightman functions given by

$$
D^{( \pm)}(x-y)=\int \frac{d^{2} k}{(2 \pi)^{2}} 2 \pi \theta\left(k^{0}\right) \delta\left(k^{2}\right) e^{\mp i k \cdot(x-y)}
$$

We have taken into account that

$$
\Delta(x-y ; \mu)=i \theta\left(x^{0}-y^{0}\right) D^{(+)}(x-y)+i \theta\left(y^{0}-x^{0}\right) D^{(-)}(x-y) .
$$

According to Wightman and Streater [18] and Coleman [19], we can define the wave functions of the states

$$
\begin{aligned}
\left|h ; j^{0}\right\rangle & =\int d^{2} x h(x) j^{0}(x)|0\rangle, \\
\left|h ; j^{1}\right\rangle & =\int d^{2} x h(x) j^{1}(x)|0\rangle,
\end{aligned}
$$

where $h(x)$ is the test function from the Schwartz class $h(x) \in \mathcal{S}\left(\mathbb{R}^{2}\right)[18$.

The norms of the states (B-5) are equal to [18, 19]

$$
\begin{aligned}
& \left\langle j^{0} ; h \mid h ; j^{0}\right\rangle=\iint d^{2} x d^{2} y h^{*}(x)\left\langle 0\left|j^{0}(x) j^{0}(y)\right| 0\right\rangle h(y)= \\
& =\frac{\bar{\eta}}{\pi} \frac{1}{\left(1+\bar{\xi} \frac{g}{\pi}\right)\left(1+(\bar{\xi}-\bar{\eta}) \frac{g}{\pi}\right)} \int \frac{d^{2} k}{(2 \pi)^{2}} 2 \pi\left(k^{0}\right)^{2} \theta\left(k^{0}\right) \delta\left(k^{2}\right)|\tilde{h}(k)|^{2}, \\
& \left\langle j^{1} ; h \mid h ; j^{1}\right\rangle=\frac{\bar{\eta}}{\pi} \frac{1}{\left(1+\bar{\xi} \frac{g}{\pi}\right)\left(1+(\bar{\xi}-\bar{\eta}) \frac{g}{\pi}\right)} \iint d^{2} x d^{2} y h^{*}(x)\left\langle 0\left|j^{1}(x) j^{1}(y)\right| 0\right\rangle h(y)= \\
& =\frac{\bar{\eta}}{\pi} \frac{1}{\left(1+\bar{\xi} \frac{g}{\pi}\right)\left(1+(\bar{\xi}-\bar{\eta}) \frac{g}{\pi}\right)} \int \frac{d^{2} k}{(2 \pi)^{2}} 2 \pi\left(k^{0}\right)^{2} \theta\left(k^{0}\right) \delta\left(k^{2}\right)|\tilde{h}(k)|^{2},
\end{aligned}
$$

where $\tilde{h}(k)$ is the Fourier transform of the test function $h(x)$. Since the norms of the states (B-5) should be positive, we get the constraint

$$
\bar{\eta}\left(1+\bar{\xi} \frac{g}{\pi}\right)\left(1+(\bar{\xi}-\bar{\eta}) \frac{g}{\pi}\right)>0 .
$$

This assumes that $\bar{\eta} \neq 0$. For $\bar{\eta}$, constrained by the requirement of the renormalizability of the massless Thirring model (4.5), the inequality (B-7) reduces to the form

$$
-g\left(1+\bar{\xi} \frac{g}{\pi}\right)>0
$$


This inequality is fulfilled for

$$
g>0,1+\bar{\xi} \frac{g}{\pi}<0 \quad, \quad g<0,1+\bar{\xi} \frac{g}{\pi}>0 .
$$

Using the vacuum expectation value of the two-point correlation function of the vector currents (B-1) we can calculate the equal-time commutator $\left[j^{0}(x), j^{1}(y)\right]_{x^{0}=y^{0}}$ and the Schwinger term. We get

$$
\begin{aligned}
{\left[j^{0}(x), j^{1}(y)\right]_{x^{0}=y^{0}} } & =-c i \frac{\partial}{\partial x^{1}} \delta\left(x^{1}-y^{1}\right)= \\
& =-\frac{\bar{\eta}}{\pi} \frac{1}{\left(1+\bar{\xi} \frac{g}{\pi}\right)\left(1+(\bar{\xi}-\bar{\eta}) \frac{g}{\pi}\right)} i \frac{\partial}{\partial x^{1}} \delta\left(x^{1}-y^{1}\right)
\end{aligned}
$$

with the Schwinger term $c$ equal to

$$
c=\frac{\bar{\eta}}{\pi} \frac{1}{\left(1+\bar{\xi} \frac{g}{\pi}\right)\left(1+(\bar{\xi}-\bar{\eta}) \frac{g}{\pi}\right)} .
$$

Due to the constraint (B-7) it is always positive. For $\bar{\eta}=1$ our expression (B-10) for the equal-time commutator coincides with that obtained by Hagen [4].

Using (B-10) we can analyse the Bjorken-Johnson-Low (BJL) limit for the Fourier transform of the two-point correlation function of the vector currents [20, 21. Following [21], we consider the Fourier transform

$$
T_{\mu \nu}(q)=i \int d^{2} x e^{i q \cdot x}\left\langle A\left|\mathrm{~T}\left(j_{\mu}(x) j_{\nu}(0)\right)\right| B\right\rangle
$$

where $q=\left(q^{0}, q^{1}\right)$ and $|A\rangle$ and $|B\rangle$ are quantum states [21]. In our case these are vacuum states $|A\rangle=|B\rangle=|0\rangle$. This gives

$$
T_{\mu \nu}(q)=i \int d^{2} x e^{i q \cdot x}\left\langle 0\left|\mathrm{~T}\left(j_{\mu}(x) j_{\nu}(0)\right)\right| 0\right\rangle .
$$

According to the BJL theorem [20, 21], in the limit $q^{0} \rightarrow \infty$ the r.h.s of Eq.(B-12) behaves as follows [21]

$$
\begin{aligned}
T_{\mu \nu}(q)= & -\frac{1}{q^{0}} \int_{-\infty}^{+\infty} d x^{1} e^{-i q^{1} x^{1}}\left\langle 0\left|\left[j_{\mu}\left(0, x^{1}\right), j_{\nu}(0)\right]\right| 0\right\rangle \\
& -\frac{i}{\left(q^{0}\right)^{2}} \int_{-\infty}^{+\infty} d x^{1} e^{-i q^{1} x^{1}}\left\langle 0\left|\left[\partial_{0} j_{\mu}\left(0, x^{1}\right), j_{\nu}(0)\right]\right| 0\right\rangle+O\left(\frac{1}{\left(q^{0}\right)^{3}}\right)
\end{aligned}
$$

For the time--space component of the two-point correlation function we get

$$
\begin{aligned}
T_{01}\left(q^{0}, q^{1}\right)= & -\frac{1}{q^{0}} \int_{-\infty}^{+\infty} d x^{1} e^{-i q^{1} x^{1}}\left\langle 0\left|\left[j_{0}\left(0, x^{1}\right), j_{1}(0)\right]\right| 0\right\rangle \\
& -\frac{i}{\left(q^{0}\right)^{2}} \int_{-\infty}^{+\infty} d x^{1} e^{-i q^{1} x^{1}}\left\langle 0\left|\left[\partial_{0} j_{0}\left(0, x^{1}\right), j_{1}(0)\right]\right| 0\right\rangle+O\left(\frac{1}{\left(q^{0}\right)^{3}}\right)
\end{aligned}
$$


Using (B-10) for the BJL limit of $T_{01}\left(q^{0}, q^{1}\right)$ we obtain

$$
T_{01}\left(q^{0}, q^{1}\right)=\frac{q^{1}}{q^{0}} \frac{\bar{\eta}}{\pi} \frac{1}{\left(1+\bar{\xi} \frac{g}{\pi}\right)\left(1+(\bar{\xi}-\bar{\eta}) \frac{g}{\pi}\right)}+O\left(\frac{1}{\left(q^{0}\right)^{3}}\right) .
$$

For $\bar{\eta}=1$ this reproduces the result which can be obtained using Hagen's solution 44. One can show that due to conservation of vector current the term proportional to $1 / q_{0}^{2}$ vanishes. The asymptotic behaviour of the Fourier transform of the two-point correlation function of the vector currents places no additional constraints on the parameters $\bar{\xi}$ and $\bar{\eta}$.

The inequality (B-7) leads to the following interesting consequences. According to Coleman [14], the coupling constant $\beta^{2}$ of the sine-Gordon model is related to the coupling constant $g$ of the Thirring model as

$$
\frac{\beta^{2}}{8 \pi}=\frac{1}{2}+d_{(\bar{\psi} \psi)^{2}}(g)=\frac{1}{2}\left(1-\frac{g}{\pi} \frac{1}{1+\bar{\xi} \frac{g}{\pi}}\right) .
$$

Hence, for the constraint $(\underline{B}-9)$ the coupling constant $\beta^{2}$ is of order $\beta^{2} \sim 8 \pi$. A behaviour and renormalizability of the sine-Gordon model for the coupling constants $\beta^{2} \sim 8 \pi$ has been investigated in [15].

\section{References}

[1] W. Thirring, Ann. Phys. (N.Y.) 3, 91 (1958).

[2] K. Johnson, Nuovo Cim. 20, 773 (1961).

[3] F. L. Scarf and J. Wess, Nuovo Cim. 26, 150 (1962).

[4] C. R. Hagen, Nuovo Cim. B 51, 169 (1967).

[5] B. Klaiber, in Lectures in theoretical physics, Lectures delivered at the Summer Institute for Theoretical Physics, University of Colorado, Boulder, 1967, edited by A. Barut and W. Brittin, Gordon and Breach, New York, 1968, Vol. X, part A, pp.141-176.

[6] K. Furuya, Re. E. Gamboa Saravi and F. A. Schaposnik, Nucl. Phys. B 208, 159 (1982).

[7] C. M. Naón, Phys. Rev. D 31, 2035 (1985).

[8] R. Roskies and F. A. Schaposnik, Phys. Rev. D 23, 558 (1981); R. E. Gamboa Saravi, F. A. Schaposnik, and J. E. Solomin, Nucl. Phys. B 153, 112 (1979); R. E. Gamboa Savari, M. A. Muschetti, F. A. Schaposnik, and J. E. Solomin, Ann. of Phys. (N.Y.) 157, 360 (1984); O. Alvarez, Nucl. Phys. B 238, 61 (1984); H. Dorn, Phys. Lett. B 167, 86 (1986).

[9] K. Harada, H. Kubota, and I. Tsutsui, Phys. Lett. B 173, 77 (1986).

[10] R. Jackiw and R. Rajaraman, Phys. Rev. Lett. 54, 1219 (1985). 
[11] G. A. Christos, Z. Phys. C 18, 155 (1983); Erratum Z. Phys. C 20, 186 (1983); A. Smailagic and R. E. Gamboa-Saravi, Phys. Lett. B 192, 145 (1987); R. Banerjee, Z. Phys. C 25, 251 (1984); T. Ikehashi, Phys. Lett. B 313, 103 (1993).

[12] R. Jackiw, Phys. Rev. D 3, 2005 (1971).

[13] J. C. Collins, in Renormalization, Cambridge University Press, Cambridge, 1984.

[14] S. Coleman, Phys. Rev. D 11, 2088 (1975).

[15] D. Amit, Y. Y. Goldschmidt, and G. Grinstein, J. Phys. A 13, 585 (1980); K. Huang and J. Polonyi, Int. Mod. Phys. A 6, 409 (1991); Zs. Gulácsi and M. Gulácsi, Adv. Phys. 47, 1 (1998); I. Nándori, J. Polonyi, and K. Sailer, Phys. Rev. D 63, 045022 (2001); Philos. Mag. B 81, 1615 (2001); G. von Gersdorf and C. Wetterich, Phys. Rev. B 64, 054513 (2001); I. Nándori, K. Sailer, U. D. Jentschura, and G. Soff, J. Phys. G 28, 607 (2002); M. Faber and A. N. Ivanov, J. Phys. A 36, 7839 (2003) and references therein. I. Nándori, K. Sailer, U. D. Jentschura, and G. Soff, Phys. Rev. D 69, 025004 (2004); H. Bozkaya, M. Faber, A. N. Ivanov, M. Pitschmann, J. Phys. A: Math. Gen. 39, 2177 (2006).

[16] J. Schwinger, Phys. Rev. 128, 2425 (1962).

[17] A. H. Mueller and T. L. Trueman, Phys. Rev. D 4, 1635 (1971); M. Gomes and J. H. Lowenstein, Nucl. Phys. B 45, 252 (1972). (2001).

[18] R. F. Streater and A. S. Wightman, in PCT, spin and statistics, Princeton University Press, Princeton and Oxford, Third Edition, 1980.

[19] S. Coleman, Comm. Math. Phys. 31, 259 (1973).

[20] J. D. Bjorken, Phys. Rev. 148, 1467 ¡81966); K. Johnson and F. E. Low, Progr. Theor. Phys., Suppl. 37-38, 74 (1966).

[21] V. De Alfaro, S. Fubini, G. Furlan, and C. Rossetti, in Currents in hadronic physics, North-Holland Publishing Co., Amsterdam • London, 1973, 